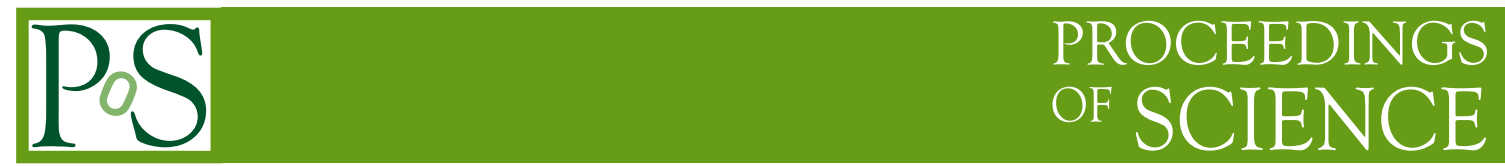

\title{
Estimating domain wall fermion chiral symmetry breaking
}

\section{Norman Christ* Columbia University}

E-mail: nhc@phys.columbia.edu

\section{RBC and UKQCD Collaborations}

The effects of explicit chiral symmetry breaking for domain wall fermions resulting from the finite size of the fifth dimension are discussed. The relation between the eigenfunctions of the five-dimensional transfer matrix and the residual mass are be reviewed and their effect on the operator mixing appearing in the calculation of $B_{K}$ is estimated.

XXIIIrd International Symposium on Lattice Field Theory

25-30 July 2005

Trinity College, Dublin, Ireland

*Speaker. 


\section{Introduction}

The development of methods for treating lattice fermions which preserve the chiral symmetries present in the continuum theory represents a major advance, permitting familiar quantities such as $B_{K}$ to be studied with improved precision and new quantities, such as $\mathrm{CP}$ violation in $\mathrm{K}$ meson decays, to be treated using lattice methods for essentially the first time $[1,2,3]$. These methods require significantly more computer resources than the standard Wilson or staggered formulations.

Calculations using the five-dimensional, domain wall fermion (DWF) formulation are more demanding by approximately a factor of $L_{s}$, the extent of the lattice in the fifth dimension. The precision of the resulting chiral symmetry depends on the separation between the left- and righthanded light degrees of freedom which are bound to the four dimensional boundaries $s=0$ and $s=L_{s}-1$, where $s$ is the fifth-dimensional coordinate. For finite $L_{s}$, propagation between these two boundaries is possible and introduces a residual breaking of chiral symmetry. Given the strong motivation to use a relatively small value of $L_{s}$ to reduce computational cost, it is important to be able to estimate the size of these residual chiral symmetry breaking effects and their dependence on $L_{s}$. This chiral symmetry breaking propagation across the fifth-dimension of the DWF lattice can be conveniently investigated by using the transfer matrix formalism, developed for the case of domain wall fermions in Ref. [4].

\section{DWF transfer matrix}

Lüscher's original discussion of the Wilson fermion transfer matrix has been exploited to analyze lattice chiral fermions in Refs. [5, 4]. The case of DWF has been thoroughly treated in Ref. [4]. One first defines a matrix $\mathscr{T}$ which, like the Wilson Dirac operator, acts on four-dimensional Dirac wave-functions (for a $L^{3} \times T$ lattice with three colors and a single flavor, this is an $L^{3} \times T \times 12$ dimensional space):

$$
\mathscr{T}=\left(\begin{array}{cc}
B^{-1} & B^{-1} C \\
C^{\dagger} B^{-1} & C^{\dagger} B^{-1} C+B,
\end{array}\right) \equiv e^{-H_{T}}
$$

where

$$
\begin{aligned}
& B_{n, m}=(5-M) \delta_{n, m}-\frac{1}{2} \sum_{\mu}\left[\delta_{n+\hat{\mu}, m} U_{n, \mu}+\delta_{n-\mu, m} U_{m, \mu}^{\dagger}\right] \quad \text { and } \\
& C_{n, m}=\frac{1}{2} \sum_{\mu}\left[\delta_{n+\hat{\mu}, m} U_{n, \mu}-\delta_{n-\mu, m} U_{m, \mu}^{\dagger}\right] \sigma_{\mu}
\end{aligned}
$$

This matrix can then be used to construct a "second-quantized" transfer matrix $T$ with acts on a $2^{L^{3} \times T \times 12}$-dimensional Fock space:

$$
T=e^{-\sum_{n, m} q^{\dagger}\left(x_{n}\right)\left(H_{T}\right)_{n, m} q\left(x_{m}\right)} .
$$

Here $q\left(x_{n}\right)$ is the quantized lattice fermion field, a function of space and time. Continuing to describe the results of Ref. [4], we can use this matrix to reproduce the usual discrete DWF path integral that is evaluated numerically in a lattice calculation $\left(L_{s}>s \geq s^{\prime} \geq 0\right)$ :

$$
\int d[\Psi(x, s)] d[\bar{\Psi}(x, s)] e^{-S_{F}} \Psi\left(x_{n}, s\right) \bar{\Psi}\left(x_{n^{\prime}}, s^{\prime}\right)=Z \operatorname{tr}\left\{T^{L_{s}-s} q\left(x_{n}\right) T^{s-s^{\prime}} q\left(x_{n^{\prime}}\right)^{\dagger} T^{s^{\prime}} \mathscr{O}\left(m_{f}\right)\right\} .
$$


Here, to be concrete, we examine the case of the calculation of a 5-dimensional fermion propagator. This expression is valid even before an average over gauge fields is performed. The Grassmann variables $\Psi$ and $\Psi$ correspond to the five-dimensional DWF fields and the operator $\mathscr{O}\left(m_{f}\right)$ represents the $m_{f}$-dependent boundary conditions imposed on the DWF path integral:

$$
\mathscr{O}\left(m_{f}\right)=\prod_{n}\left(\hat{c}_{n} \hat{c}_{n}^{\dagger}+m_{f} \hat{c}_{n}^{\dagger} \hat{c}_{n}\right)\left(\hat{d}_{n} \hat{d}_{n}^{\dagger}+m_{f} \hat{d}_{n}^{\dagger} \hat{d}_{n}\right)
$$

where $\hat{c}_{n}$ and $\hat{d}_{n}^{\dagger}$ are the right and left-handed components of $q\left(x_{n}\right)$. When $m_{f}=0, \mathscr{O}\left(m_{f}=0\right)$ projects onto a "surface" vacuum state $\left|0_{S}\right\rangle$ which is annihilated by $\hat{c}_{n}$ and $\hat{d}_{n}$.

We can analyze the large $L_{s}$ behavior of Eq. 2.5 by introducing a new Fock space basis using the eigenfunctions of $H_{T}: H_{T} \psi_{k}^{ \pm}= \pm E_{k}^{ \pm} \psi_{k}^{ \pm}$and expressing the original fermionic operator $q\left(x_{n}\right)$ as:

$$
q\left(x_{n}\right)=\sum_{k} \psi_{k}^{+}\left(x_{n}\right) \hat{o}_{k}+\sum_{k} \psi_{k}^{-}\left(x_{n}\right) \hat{p}_{k}^{\dagger}
$$

Using these operators and dropping an over-all factor, the transfer matrix of Eq. 2.4 becomes:

$$
T=e^{-\left(\sum_{k} E_{k}^{+} \hat{o}_{k}^{\dagger} \hat{o}_{k}+\sum_{k} E_{k}^{-} \hat{p}_{k}^{\dagger} \hat{p}_{k}\right)}
$$

It is now easy to discuss the large-s behavior of the transfer matrix $T^{s}$ :

$$
T^{s} \approx\left|0_{T}\right\rangle\left\langle 0_{T}\left|+\sum_{k} e^{-s E_{k}^{+}} \hat{o}_{k}^{\dagger}\right| 0_{T}\right\rangle\left\langle 0_{T}\left|\hat{o}_{k}+\sum_{k} e^{-s E_{k}^{-}} \hat{p}_{k}^{\dagger}\right| 0_{T}\right\rangle\left\langle 0_{T}\right| \hat{p}_{k}+\ldots
$$

Here the "transfer matrix" vacuum $\left|0_{T}\right\rangle$ is annihilated by $\hat{o}_{k}$ and $\hat{p}_{k}$. In the limit of large $s, T^{s}$ will simply project onto $\left|0_{T}\right\rangle$. The next leading corrections in Eq. 2.9 come by occupying one of the fermionic states corresponding to eigenvectors of $H_{T}$ with eigenvalue $E_{k}^{ \pm}$close to zero.

While there are few exact results regarding those eigenfunction of the operator $H_{T}$ with small eigenvalues, it is likely that they fall into two classes $[6,7,8,9]$ : the first is possibly rare, localized states with eigenvalues approaching zero while the second is more numerous de-localized or extended states whose eigenvalues lie above a mobility edge $\lambda_{c}, E_{k}^{ \pm} \geq \lambda_{c}[9]$.

\section{Residual mass}

We can now combine Eq. 2.9 with this assumed eigenspectrum of $H_{T}$ to estimate the dependence of residual DWF chiral symmetry breaking on $L_{s}$. We start by examining a general matrix element of a product of "physical" 4-dimension fermion fields which is best represented by a product of operators containing right-handed and left-handed fermion fields, evaluated on the $s=L_{s}-1$ and $s=0$ boundaries: $O_{R}$ and $O_{L}$ respectively. Generalizing Eq. 2.5 and, for simplicity, setting $m_{f}=0$ we find:

$$
\begin{aligned}
\left\langle O_{R} O_{L}\right\rangle=\left\langle 0_{S}\left|O_{R} T^{L_{s}} O_{L}\right| 0_{S}\right\rangle \approx & \left\langle 0_{S}\right| O_{R}\left\{\left|0_{T}\right\rangle\left\langle 0_{T}\left|+\sum_{k} e^{-L_{s} E_{k}^{+}} \hat{o}_{k}^{\dagger}\right| 0_{T}\right\rangle\left\langle 0_{T}\right| \hat{o}_{k}\right. \\
& \left.+\sum_{k} e^{-L_{s} E_{k}^{-}} \hat{p}_{k}^{\dagger}\left|0_{T}\right\rangle\left\langle 0_{T}\right| \hat{p}_{k}+\ldots\right\} O_{L}\left|0_{S}\right\rangle .
\end{aligned}
$$

Here the leading term, the projection operator $\left|0_{T}\right\rangle\left\langle 0_{T}\right|$, divides the Greens function into two independent factors demonstrating the separate, flavored chiral symmetry of the left- and right-handed 
fermions. The first correction permits quark number \pm 1 exchanges between these two otherwise independent sectors. This suggests that the second and third terms in Eq. 3.1 should be interpreted at low energies as the residual mass operator $m_{\mathrm{res}} \bar{\psi} \psi$ expressed in this 5-dimensional language.

A relation between this residual mass term and the second and third terms in the operator in curly brackets in Eq. 3.1 can be obtained if we express the operators $o_{k}$ and $p_{k}$ in terms of the conventional 4-dimension field $q(x)$, inverting Eq. 2.7:

$$
\begin{aligned}
\sum_{n} m_{\mathrm{res}} \bar{\psi}\left(x_{n}\right) \psi\left(x_{n}\right) \rightarrow & m_{\mathrm{res}} \sum_{n}\left\{q_{R}\left(x_{n}\right)^{\dagger}\left|0_{T}\right\rangle\left\langle 0_{T}\left|q_{L}\left(x_{n}\right)-q_{R}\left(x_{n}\right)\right| 0_{T}\right\rangle\left\langle 0_{T}\right| q_{L}\left(x_{n}\right)^{\dagger}+\right\} \\
\stackrel{?}{\approx} & \sum_{n, n^{\prime}} \sum_{k} \psi_{k}^{+}\left(x_{n}\right) \psi_{k}^{+}\left(y_{n^{\prime}}\right)^{\dagger} e^{-L_{s} E_{k}^{+}} q_{R}\left(x_{n}\right)^{\dagger}\left|0_{T}\right\rangle\left\langle 0_{T}\right| q_{L}\left(y_{n^{\prime}}\right) \\
& +\sum_{n, n^{\prime}} \sum_{k} \psi_{k}^{-}\left(x_{n}\right)^{\dagger} \psi_{k}^{-}\left(y_{n^{\prime}}\right) e^{-L_{s} E_{k}^{-}} q_{R}(x)\left|0_{T}\right\rangle\left\langle 0_{T}\right| q_{L}(y)^{\dagger} .
\end{aligned}
$$

Here the RHS of Eq. 3.2 is the residual mass term written in the transfer matrix language while the expression in Eq. 3.3 is a rewritten version of the leading order contribution from the second and third terms in Eq. 3.1.

We can justify this relation and estimate $m_{\text {res }}$, if we assume that the quantities $\sum_{k} \psi_{k}^{ \pm}\left(x_{n}\right) \psi_{k}^{ \pm}\left(y_{n^{\prime}}\right)$ are localized on the long distance scale at which $m_{\text {res }}$ is defined:

$$
K_{a, b}(x, y)=\sum_{k, a} \psi_{k, a}^{ \pm}(x)^{\dagger} \psi_{k, b}^{ \pm}(y) e^{-L_{s} E_{k}^{ \pm}} \approx m_{\mathrm{res}} \delta^{4}(x-y) \delta_{a, b},
$$

where $a$ and $b$ are spin-color indices and we assume that the diagonal spin-color structure will appear and any dependence on the label \pm will disappear when a volume and/or gauge average is performed. We can then approximate: $m_{\mathrm{res}}=\frac{1}{12} \int d^{4} x K_{a, a}(x, y) \approx \frac{1}{12} R^{4} K_{a, a}(y, y)$. Were the "radius" $R$ estimates the small region in $x$ that contributes and a sum over the repeated index $a$ is intended. Finally, $K(y, y)$ and hence $m_{\text {res }}$ can be determined by integrating over $y$ and using the orthonormality of the eigenfunctions $\psi_{k, a}^{ \pm}(y)$ :

$$
\begin{aligned}
m_{\mathrm{res}} & =\frac{R^{4}}{12} K_{a, a}(y, y)=\frac{R^{4}}{12 L^{4}} \int d^{4} y K_{a, a}(y, y) \\
& =\frac{R^{4}}{12 L^{4}} \sum_{k} e^{-L_{s} E_{k}^{ \pm}}=R^{4} \int_{o}^{\infty} d \lambda \rho(\lambda) e^{-L_{s} \lambda} \\
& \sim R_{e}^{4} \rho_{e}\left(\lambda_{c}\right) \frac{e^{-\lambda_{c} L_{s}}}{L_{s}}+R_{l}^{4} \rho_{l}(0) \frac{1}{L_{s}} .
\end{aligned}
$$

Here $\rho(\lambda)$ on the RHS of Eq. 3.6 is the density of eigenvalues of $H_{T}$ per unit space-time volume, color and spin. The final Eq. 3.7 is a generalization of Eq. 3.6 displaying the expected contributions of localized $(l)$ and extended ( $e$ ) modes with possibly different average sizes $R_{l}$ and $R_{e}$. In this final equation we have also taken the limit of large $L_{s}$. This is the "standard" result for the dependence of $m_{\text {res }}$ on $L_{s}$, although the factor of $1 / L_{s}$ is usually missing from the left-most term.

\section{Operator mixing}

Having developed a reasonably systematic approach to estimating the size of DWF chiral symmetry breaking, we can now apply this method to a quantity of special interest: the mixing 
between the usual $O_{L L}$ operator whose matrix element gives $B_{K}$ and potentially larger operators whose matrix elements do not vanish in the chiral limit. Here we will examine the case of mixing between $O_{L L}$ and $O_{L R}$ where:

$$
O_{L L / L R}=\bar{s} \gamma^{\mu}\left(1-\gamma^{5}\right) d \bar{s} \gamma^{\mu}\left(1 \pm \gamma^{5}\right) d
$$

The mixing between these two operators can be represented by the equation $O_{L L}^{\text {cont }}=Z_{L L, L L} O_{L L}+$ $Z_{L L, L R} O_{L R}$, expressing the continuum operator $O_{L L}^{\text {cont }}$ in terms of those defined on the lattice. The mixing term $Z_{L L, R R}$ is required to remove the contribution of the wrong chirality operator $O_{L R}$ that will appear in matrix elements of the lattice operator $O_{L L}$ coming from residual chiral symmetry breaking.

The mixing coefficient of interest can be computed by evaluating a Greens function far offshell to avoid innocuous "mixing" caused by low-energy, spontaneous, chiral symmetry breaking:

$$
\begin{aligned}
Z_{L L, L R}(\mu) & =\frac{1}{128} \prod_{1 \leq i \leq 4}\left\{\int d^{4} x_{i} e^{i p_{i} \cdot x_{i}}\right\}\left\langle 0\left|\bar{d}_{R}\left(x_{1}\right) \not p_{1} \gamma^{\mu} \not p_{2} s_{R}\left(x_{2}\right) O_{L L}(0) \bar{d}_{L}\left(x_{3}\right) \not p_{3} \gamma^{\mu} \not p_{4} s_{L}\left(x_{4}\right)\right| 0\right\rangle_{p_{i}^{2}=\mu^{2}} \\
& \approx \frac{1}{8} \prod_{1 \leq i \leq 2}\left\{\int d^{4} x_{i} e^{i p_{i} \cdot x_{i}}\right\}\left\langle 0\left|\bar{d}_{R}\left(x_{1}\right) \not p_{1} \gamma^{\mu} \not p_{2} s_{R}\left(x_{2}\right) \bar{s}_{L}(0) \gamma^{\mu} d_{L}(0)\right| 0\right\rangle_{p_{i}^{2}=\mu^{2}}
\end{aligned}
$$

In the top part of Eq. 4.2 we show the usual off-shell Greens function that will determine the $O_{L R}$ component present in the $O_{L L}$ operator. This has been simplified in the lower equation by removing the two normal, chirality conserving fermion contractions - a step accurate only at tree level.

We next evaluate the RHS of Eq. 4.2 using the method discussed above. In the transfer matrix language, the expression to be evaluated takes the form

$$
Z_{L L, L R}(\mu) \approx \frac{1}{8} \prod_{1 \leq i \leq 2}\left\{\int d^{4} x_{i} e^{i p_{i} \cdot x_{i}}\right\}\left\langle 0_{S}\left|q_{R}^{d^{\dagger}}\left(x_{1}\right) \not p_{1} \gamma^{\mu} \not p_{2} q_{R}^{s}\left(x_{2}\right) T^{L_{s}} q_{L}^{s \dagger}(0) \gamma^{\mu} q_{L}^{d}(0)\right| 0_{S}\right\rangle_{p_{i}^{2}=\mu^{2}}
$$

We can now insert the large-s expansion for $T^{s}$ of Eq. 2.9 into Eq. 4.3. Clearly the leading order term $\left|0_{T}\right\rangle\left\langle 0_{T}\right|$ does not contribute since the state $\left|0_{T}\right\rangle$ is an $S U(3)_{L} \times S U(3)_{R}$ singlet while the operator $q_{L}^{s \dagger}(0) q_{L}^{d}(0)$ transforms as an $(8,1)$ under this group. Likewise the contribution from a single state of the sort represented by the second and third terms in Eq. 2.9 corresponds to the $(3,1)$ representation and hence also vanishes.

Thus, we must go to the next term in the large $s$ expansion of $T^{s}$, one containing two filled eigenstates of $H_{T}$. This would suggest a double suppression of order $m_{\mathrm{res}}^{2}$. However, since two flavors of quarks are involved, there may be a larger contribution from a gauge configuration that supports a single localized mode with near-zero eigenvalue of $H_{T}$ [10]. While such a mode is suppressed (it will appear infrequently in the gauge average and must be located near the position of the weak operator) if this mode can be used twice, once for the $s$ quark and once for the $d$ quark, only a single suppression factor will be involved. However, this is not possible here: the product $q_{L}^{s \dagger}(0) q_{L}^{d}(0)$ has quark number zero while the pairs $\hat{o}_{k}^{s} \hat{o}_{k}^{d}$ and $\hat{p}_{k}^{s} \hat{p}_{k}^{d}$ have quark number \pm 2 .

Thus, a non-zero contribution to the leading term in Eq. 4.3, requires two independent eigenfunctions $\psi_{k}^{+}$and $\psi_{k^{\prime}}^{-}$for the $s$ and $\bar{d}$ quarks respectively. Carrying out a heuristic estimate, similar 
to that in Eqs. 3.4-3.7, gives:

$$
\begin{aligned}
Z_{L L, R R} & \approx \int_{0}^{\infty} d \lambda^{+} d \lambda^{-} e^{-s\left(\lambda^{+}+\lambda^{-}\right)}\left\{R_{e}^{8} \rho_{e e}\left(\lambda^{+}, \lambda^{-}\right)+R_{e}^{4} R_{l}^{4} \rho_{e l}\left(\lambda^{+}, \lambda^{-}\right)+R_{l}^{8} \rho_{l l}\left(\lambda^{+}, \lambda^{-}\right)\right\} \\
& \approx\left\{R_{e}^{4} \rho_{e}\left(\lambda_{c}\right) \frac{e^{-\lambda_{c} L_{s}}}{L_{s}}+R_{l}^{4} \rho_{l}(0) \frac{1}{L_{s}}\right\}^{2}=m_{\mathrm{res}}^{2} .
\end{aligned}
$$

where, for example, $\rho_{l l}\left(\lambda^{+}, \lambda^{-}\right)$is the joint distribution of localized, positive and negative energy modes with eigenvalues $\lambda^{+}$and $\lambda^{-}$. One obtains the lower line of Eq. 4.4 by approximating these joint densities by products of single eigenvalue densities and taking the limit of large $L_{s}$.

\section{Conclusion}

Using the transfer matrix formalism of Furman and Shamir we have made rough estimates of the large $L_{S}$ behavior of the DWF residual mass and the chiral symmetry violating mixing of the four-Fermi operators which contribute to a calculation of $K^{0}-\bar{K}^{0}$ mixing. We find that this operator mixing, which changes chirality by two units, is doubly suppressed, behaving as $m_{\mathrm{res}}^{2}$.

\section{Acknowledgments}

Helpful discussions with Peter Boyle, Chris Dawson, Robert Mawhinney and other members of the RBC and UKQCD collaborations are gratefully acknowledged. This work was supported by DOE grant DE-FG02-92ER40699.

\section{References}

[1] P. M. Vranas, Domain wall fermions and applications, Nucl. Phys. Proc. Suppl. 94 (2001) 177-188, [arXiv: hep-lat/0011066].

[2] P. Hernandez, Ginsparg-wilson fermions: Practical aspects and applications, Nucl. Phys. Proc. Suppl. 106 (2002) 80-85, [hep-lat/0110218].

[3] L. Giusti, Exact chiral symmetry on the lattice: Qcd applications, hep-lat/0211009.

[4] V. Furman and Y. Shamir, Axial symmetries in lattice 'qcd' with kaplan fermions, Nucl. Phys. B439 (1995) 54-78, [hep-lat/9405004].

[5] R. Narayanan and H. Neuberger, Chiral determinant as an overlap of two vacua, Nucl. Phys. B412 (1994) 574-606, [hep-lat/9307006].

[6] R. G. Edwards, U. M. Heller, and R. Narayanan, Spectral flow, chiral condensate and topology in lattice qcd, Nucl. Phys. B535 (1998) 403, [hep-lat/9802016].

[7] S. Aoki and Y. Taniguchi, Chiral properties of domain-wall fermions from eigenvalues of 4-dimensional wilson-dirac operator, Phys. Rev. D65 (2002) 074502, [hep-lat/ 0109022$].$

[8] P. Boyle, Localisation and chiral symmetry in 2+1 flavour domain wall qcd, PoS(LAT2005)141 (2005).

[9] M. Golterman and Y. Shamir, Localization in lattice qcd, Phys. Rev. D68 (2003) 074501, [hep-lat/0306002].

[10] M. Golterman and Y. Shamir, Before sailing on a domain-wall sea, Phys. Rev. D71 (2005) 034502, [hep-lat/0411007]. 